\title{
Interactive Effects of Age and Interface Differences on Search Strategies and Performance
}

\author{
Jessie Chin Wai-Tat Fu \\ Applied Cognitive Science Laboratory \\ University of Illinois at Urbana Champaign \\ $405 \mathrm{~N}$ Mathews Avenue \\ Urbana, IL 61801 \\ \{chin5,wfu\}@illinois.edu
}

\begin{abstract}
We present results from an experiment that studied the information search behavior of younger and older adults in a medical decision-making task. To study how different combination of tasks and interfaces influenced search strategies and decision-making outcomes, we varied information structures of two interfaces and presented different task descriptions to participants. We found that younger adults tended to use different search strategies in different combination of tasks and interfaces, and older adults tended to use the same top-down strategies across conditions. We concluded that older adults were able to perform mental transformation of medical terms more effectively than younger adults. Thus older adults did not require changing strategies to maintain the same level of performance.
\end{abstract}

\section{Author Keywords}

Age differences, web search, search strategies, cost-benefit analysis, knowledge structure, interface affordance

\section{ACM Classification Keywords}

H.3.3 Information Search and Retrieval: Search Process. H.5.2 User Interfaces: Theory and methods I.2.8 Problem solving, control methods, and search. J.3 Life and medical science: Health J.4 Social and behavioral sciences

\section{General Terms}

Experimentation

\section{INTRODUCTION}

In the United States, $75 \%$ to $80 \%$ of the Internet users look for online health information. At least $31 \%$ of the users claim that they have been significantly helped by following medical advice or health information gathered from the Internet [18]. Indeed, online health information websites have become an important source from which people gather relevant medical knowledge and healthcare information. In particular, older adults are found to be frequent consumers

Permission to make digital or hard copies of all or part of this work for personal or classroom use is granted without fee provided that copies are not made or distributed for profit or commercial advantage and that copies bear this notice and the full citation on the first page. To copy otherwise, or republish, to post on servers or to redistribute to lists, requires prior specific permission and/or a fee.

CHI 2010, April 10-15, 2010, Atlanta, Georgia, USA.

Copyright 2010 ACM 978-1-60558-929-9/10/04 ...\$10.00 of online health information given their higher demands on self-care and medication adherence.

According to the Pew report [18], one major reason why older adults seek online health information is to inform medical decision making. Although there have been studies that investigated how older adults look for health information online, few have looked at how they comprehend and interpret the health information to inform medical decisions or judgment. In this paper, we present results from an experiment that compared search behavior of younger and older adults. In the experiment, younger and older adults looked for health information on different versions of a medical website. Then they used the health information to make medical decisions and judge the likelihood of different diseases based on a certain set of symptoms. Our goals are: to understand how different presentation of health information may induce different strategies among younger and older adults as they interact with the websites, to investigate whether their final decision-making or judgment outcomes may be influenced as a result of the different strategies, and to discuss whether they may be able to acquire some medical knowledge after they interact with the sites.

Previous research found that younger and older adults tended to adopt different search strategies when they looked for information online [4]. The choice of strategies seemed to be sensitive to the task demands as well as the cognitive profiles of information seekers. For example, results showed that in ill-defined search tasks (tasks that were more open-ended and often without a clear information goal), older adults were better at finding and utilizing online information than younger adults. But in well-defined tasks (tasks that required retrieval of specific information) older adults performed much worse than younger adults [4]. One explanation was that younger and older adults could adopt different search strategies that best fit their cognitive constraints and background knowledge. Furthermore, the different search strategies would lead to differences in performance in face of different task demands. Specifically, younger adults would use more bottom-up interface-driven strategies that led them to perform better in well-defined search tasks; on the other hand, older adults would use 
more top-down knowledge-driven strategies that would lead them to perform better in the ill-defined search task. The use of a bottom-up interface-driven strategy implied that users would tend to look up more links, leave a web page quickly, and browse more within-category links. The topdown knowledge-driven strategy implied that users would tend to look up only a subset of links, take longer time to click a link and leave a web page, and browse more between-category links. Given that the information is usually organized into categories on the web, how often users switch to different categories to look up links can be a measure of the individual differences in search strategies. For the bottom-up interface-driven strategies, users would browse more links in the same category before switching to a new category. In other words, there was a higher tendency that users selected a new link in the same category to which the previous link belonged if they adopted the bottom-up interface-driven strategy. The clicking patterns would more likely depend on the organization of information shown in the interface. For the top-down knowledge-driven strategy, users may switch across different categories more often. The clicking patterns would more likely depend on their background knowledge instead of the interface. What is still unclear is whether the use of different search strategies is also sensitive to demands imposed by the search environments, such as those that are caused by different presentation formats of information on a web page. Our study aims at filling this gap by studying how different interfaces may induce different search strategies for younger and older adults, and how different strategies may lead to differential changes in their internal knowledge structures and decision-making outcomes.

We were interested in examining the effects of different interfaces and tasks on information search behavior of younger and older adults. We also measured participants' knowledge structures to understand to what extent their categorization of medical terms conformed to those imposed by the interfaces. We also compared how their knowledge structures might differ before and after the search tasks. In addition, we manipulated different kinds of tasks in terms of their compatibility with the interface, which might demand different levels of mental transformation during the search. We were interested in the following three questions:

(a) Do older adults and younger adults tend to use different strategies, such as the top-down search strategy or bottom-up search strategy?

(b) If younger and older adults do tend to use different search strategies, how will this tendency change across different interfaces and tasks? In other words, to what extent do older and younger adults use different search strategies in different interfaces and tasks?

(c) In addition to the adaptation of search strategies, how do the different search environments influence the extent to which younger and older adults can acquire new knowledge from the interaction?

\section{RELATED WORK}

More and more studies are trying to explore how older adults, the population who arguably needs health information the most, interact with the Internet for different purposes. One survey study showed that one third of the older adults turn to the Internet for health information about health care issues [9]. In addition to the research about Internet usage pattern of older adults, there was a study that showed that older adults had more difficulty searching for information on the Web in terms of efficiency and correctness [23]. The study claimed that the different search performance between younger and older adults could be explained by older adults' poorer knowledge about different features of the WWW, such as the search engine, search box, or URL [23]. There was also a study that discussed the worse search performance of older adults in terms of their decline in fluid cognitive functions [6]. In addition to these studies that focused on comparing the search performance between age groups, a qualitative study also showed that older adults tended to use different search strategies than younger adults [7]. Thus, in addition to the lack of Internet experience and cognitive deficits of older adults, results from recent research suggested that the stories behind older adults' worse search performance could be more complex than originally concluded.

Recently, researchers have conducted empirical studies to investigate how cognitive abilities and task environments influence the strategy use of different age groups and how they influenced performance in information search tasks [ 1 , $4,19]$. In [4], older adults were found to perform better in ill-defined search tasks -- tasks that were more exploratory with multiple paths to multiple solution states as well as more representative of our everyday problem [20]; while younger adults were better in the well-defined search tasks - tasks that required seeking of more specific information. One important finding was that both younger and older adults could adopt search strategies with better utilities in terms of the cognitive and knowledge constraints. Older adults tended to use more top-down knowledge-driven search strategies that required more background knowledge and less perceptual-motor operations; younger adults tended to use more bottom-up interface-driven strategies that required less background knowledge and more perceptualmotor operations. Results suggested that both younger and older adults were adaptive in their selection of strategies, in the sense that they tended to select strategies that "best fit" the constraints imposed by their cognitive profiles.

The phenomenon that older adults rely on more knowledge or crystallized ability to compensate for their decline in fluid ability has been widely found in the psychological literature. For example, one reading study suggested that older adults with varying levels of domain knowledge had different patterns of time allocation on reading the different types of texts. Specifically, older adults would spend more 
time doing integration and elaboration than younger adults. This knowledge-driven reading helped older adults create situation models during reading $[16,22]$. In problemsolving research, a study found that older adults with fewer cognitive resources tended to look up less information and rely on their treatment knowledge to make faster treatment decisions than younger adults [15]. In the decision making literature, a study showed that older adults, with their declined cognitive functions, favored using less cognitively demanding, more information-frugal strategies, as they adjusted to the non-compensatory tasks. This was taken as evidence that older adults were highly adaptive in their selection of strategies to compensate for declined cognitive abilities in decision making [14].

In the studies discussed above, researchers put more efforts on showing the adaptation of strategies by older adults to overcome their decline in fluid abilities. However, in most of the human-computer-interaction (HCI) research, the user will tend to consider a computer (or any interactive user interface) as an external knowledge repository as well as an outside computational resource [13]. Thus many argued that human and computer could be considered a coupled system with components that work together as a distributed cognitive processor [12]. Users can offload the information processing costs to the external environment to reduce computational costs [17]. According to this view, adaptation of cognitive process can be better understood in terms of how the human behavior may interact with the coupled computational system in the task environment. Indeed, there has been plenty of HCI research that showed how different task environments (e.g., interfaces) might channel the use of different strategies in multiple contexts.

One study used a map navigation task to study adaptive information seeking behavior [10]. They found that information seeking behavior was adapted to the trade-off between search costs and the utility of information. They showed that a Bayesian satisficing model was able to explain why people did not fully explore the environment, and ended up with suboptimal performance in information seeking. One related study also suggested that the label relevance and the item position could influence the menu search strategies. People tended to assess more items while the label relevance of distracters was higher regardless of the item position. However, people still adopted a cognitively easy search strategy if getting to the target item on the top of webpage [3]. Lastly, a computational cognitive model for web navigation, SNIF-ACT, used utility estimation from the information scent, link position, and adaptive link assessment (Bayesian Satisficing Model) to predict link selection and when users would leave a page. The model assumed that people adjusted their search behavior based on the trade-off between the cost of seeking information and the utility of information [11]. In sum, previous research seemed to suggest that information search strategies tended to be highly sensitive to the cost-benefit tradeoffs afforded by the task environment.
Given that information search behavior is context-sensitive, how will people react if the task environments are different? Part of the answers to this question could be found from research on skill acquisition that discussed the strategy shifts in different task environments. One study demonstrated that younger adults were able to adapt the strategies to better fit the demands of task in a simple computer skill acquisition task [5]. However, in the related aging study, research found that older adults tended to keep using the rule-based strategy or shift to a memory retrieval strategy later than younger adults [24]. However, they showed that in conditions under which there was a higher shift affordance, in which strategy shift became more beneficial, older adults would shift to a memory-based strategy more quickly. There have been a few suggested reasons for why older adults were more reluctant to shift strategies than younger adults. For example, it was possible that older adults might lack confidence in memory function or aging related learning deficits, and thus were less likely to adopt strategies that tapped on those abilities. We would like to see to what extent older adults are able to adjust their strategies in different search task environments in the current study.

\section{METHOD}

We investigated how older and younger participants used two versions of the health information interfaces that we designed as they engaged in a series of medical decision tasks. In addition, we were interested in knowing how their internal knowledge may interact with the different presentation of health information on the interfaces. Based on prior research, we designed and conducted an experiment to directly test the effects of age difference and interfaces on search and decision-making behavior.

\section{Participants}

Fifty-one participants were recruited from the community of a medium-sized city in the United States. Data from five participants were lost due to the technical problems. Of the remaining forty-six participants, twenty-three were younger participants (Age range from 18 to 28; Mean=21.3, $\mathrm{SD}=2.38$ ) and twenty-three were older participants (Age range from 60 to 77; Mean $=68.52, \mathrm{SD}=5.28$ ). Thirty participants were females (65.2\%). All participants had basic computer skills and Internet experience (used the Internet at least two times a week). Most participants had completed at least some years of college. There was no significant difference on the level of education between younger and older participants. Also, there was no age difference on their self-reported health care related experience (including reading health materials, health information searching or health related professional).

\section{Web Search Interfaces}

We created two different interfaces based on the materials from two well-known public medical information websites WebMD (http://www.webmd.com) and MedlinePlus.gov (http://medlineplus.gov). We selected about 350 disease items and 52 associated symptoms from the websites. In the 


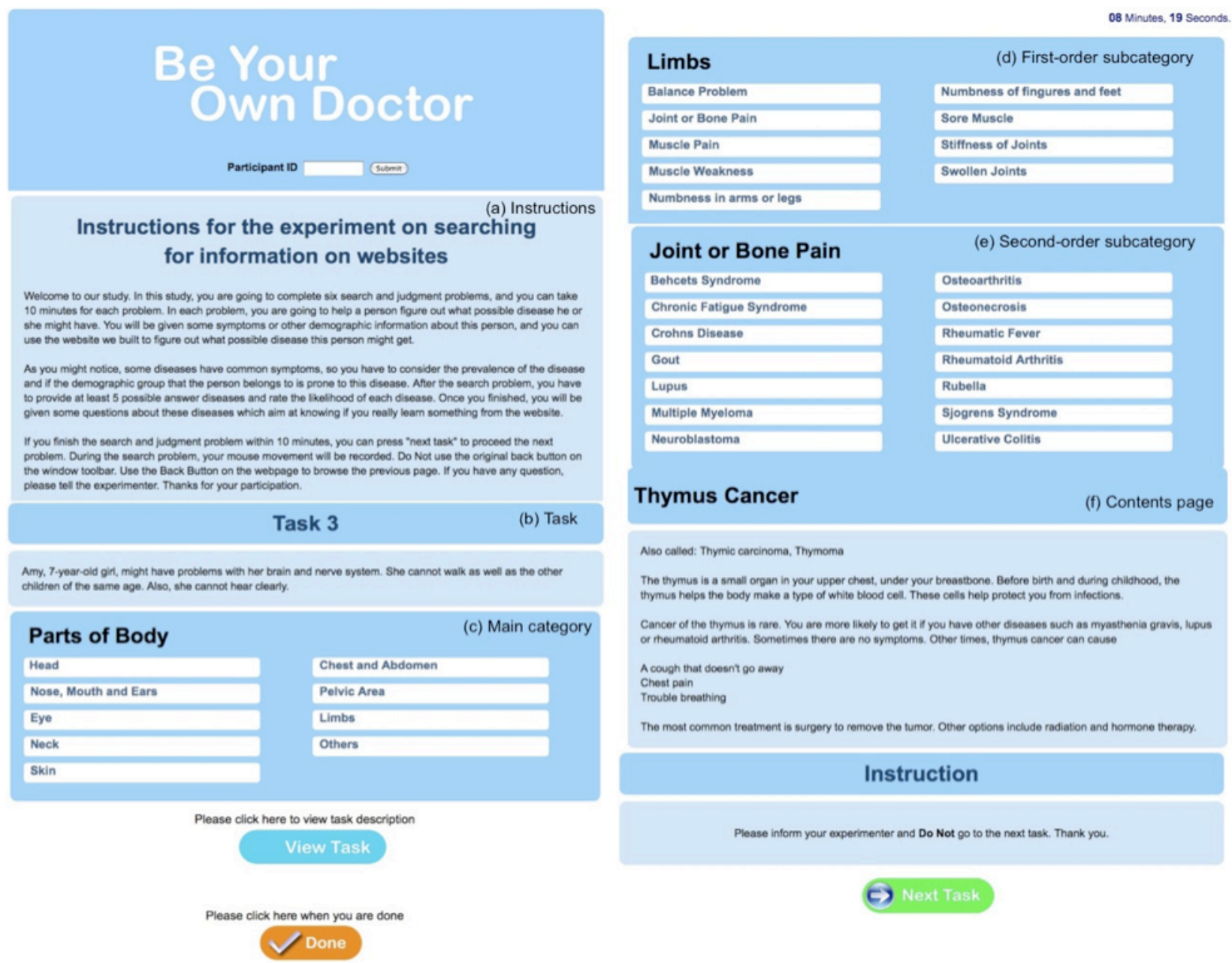

Figure 1. Example pages of the parts interface: (a) instructions, (b) task, (c) main category, (d) first-order subcategory (symptoms), (e) second-order subcategory (disease), (f) description of disease.

parts interface, we categorized these symptoms based on the body parts at which the symptoms could be found; in the systems interface, we categorized symptoms based on the functional body systems in which the symptoms occurred.

There were two main reasons for creating the two interfaces. First, these two categorizations of symptoms are the most commonly found ones in existing medical information websites. From our knowledge we could not find any prior research comparing the two. Second, we hypothesized that the nature of the two interfaces could afford two qualitatively different search strategies. For the parts interface, because participants only needed to refer to the body parts (e.g., chest pain $\rightarrow$ chest) at which the symptom occurred, it afforded a strategy that allowed a more bottom-up match of features that did not require much medical knowledge. In contrast, because the systems interface categorized symptoms by the functional systems, participants needed to utilize more medical knowledge to infer the system(s) with which the symptoms could be associated (e.g., chest pain $\rightarrow$ heart/circulation system). We therefore regarded the systems interface as one that afforded a more knowledge-driven top-down strategy.
In the parts interface, there were nine main categories: head area, nose, mouth and ears, eyes, neck area, chest and abdomen area, pelvic area, limbs, skin, and others. In the systems interface, the nine main categories were: blood, heart and circulation system, brain and nerve system, bones, joints and muscle system, digestive system, endocrine system, immune system, kidney and urinary system, breathing and nasal system, and others. These categories were all listed on the first page of the interface (Figure 1c). After participants clicked on an item on the main category, they would be taken to the symptoms page (for both interfaces), which listed symptoms that belonged to the chosen main category (a part or system). For example, as shown in Figure 1d, participants could see "muscle pain" and "joint or bone pain", etc. after clicking on the main category of "limbs". When participants clicked on a symptom, they would be taken to the disease page, on which there was a list of diseases that were associated with the chosen symptom. Participants could then click on one of the diseases to see a brief description about this disease, which would contain information about the disease definition, possible symptoms, prevalence rates, and the risk factors of this disease. 
Half of the participants from each age group performed the tasks in the parts interface, and the other half performed in the systems interface. For both interfaces, the information contents were identical, only the categorization was different. Font size and font color were complied with the rules suggested by the previous studies [8].

\section{Experimental Design}

A $2 \times 2 \times 2$ mixed factor experimental design was used. There were two between-subject variables: Age (young/old) and Interface (parts/systems - will be explained later), and one within-subject variable, Task (match with the interface VS. mismatch with the interface). We recruited two age groups, younger and older participants, to manipulate the age effects on search performance. In addition, half of the participants were assigned to perform the tasks in the parts interface and the other half in the systems interface. Hence, every participant only used one of the interfaces. Finally, all participants completed two kinds of information search tasks in their assigned interface. Their search behavior and performance were also recorded during the experiment. The detailed design is described below.

\section{Measures of Declarative and Structural Medical Knowledge}

To capture participants' background medical knowledge, we asked them to rate the prevalence rates of 18 randomly selected diseases from WebMD (http://www.webmd.com). Participants judged how often the disease occurs in the United States using a three-point Likert's Scale. We measured their accuracy by comparing their answers to the statistics from the National Institute of Health. Mean deviance scores were used as a measure of their background medical knowledge in the rest of the analysis.

We also used the card-sorting method to measure the extent to which participants' medical knowledge structures matched those that we used in the two interfaces. Twenty common symptoms were selected from our interfaces, such as coughing, difficulty breathing and chest pain. Participants were asked to put the symptom cards into piles before and after the search tasks according to their own rules. We coded the card-sorting data by transforming them to all possible 190 pairwise comparisons formed by the 20 symptoms. We then assigned 1 to the pair if two symptoms were put in the same category and 0 otherwise. The same procedure was conducted based on the categorization of the symptoms for each of the two interfaces. We then calculated the correlations between the participants' pairwise comparisons to those from each of the interfaces before and after the tasks. We called this correlation measure mental-interface match index. A high match index would imply that the mental categorizations of symptoms matched well with those in the interface. By comparing this match index before and after the task, we could also derive the extent to which their mental categorization was influenced by their interaction with the interface.

\section{Information Search task}

Participants were given six tasks in a random order. Each task described a person who had some specific symptoms. Participants had to come up with five possible diseases and give the likelihood ratings from one to three for each disease based on a 3-point Likert's scale. Participants then completed three multiple-choice questions about diseases related to the tasks. Two performance scores were measured by the correct scores of likelihood judgment ratings and the accuracy of multiple-choice questions.

There were two types of tasks. The task description for the parts tasks tended to describe symptoms by body parts, while the systems tasks tended to describe symptoms by functional systems. Here are two examples:

A parts task: Calvin, slightly overweight male, sometimes feels pain around his chest and back. He also has a slight fever recently.

A systems task: Alison feels her digestive system might have some problems. She has stomachache and diarrhea recently.

Each task type was designed to have descriptions that matched more or less the main categories in each of the interfaces. In the first example above, "the pain around his chest" in the description would provide participants a relatively direct connection to the "chest and abdomen area" in the parts interface. However, in the systems interface, chest pain could be found under the "blood, heart and circulation" system, which apparently required more top-down elaboration on the symptoms. Similarly, in the second example above, "digestive system" was one of the main categories in the systems interface. Participants could then find stomachache and diarrhea after clicking on the digestive system link. However, in the parts interface, participants needed to click on the "chest and abdomen area" for "stomachache" or the "pelvic area" for "diarrhea" to get to the possible disease.

\section{Procedure}

When the experiment began, participants were asked to perform the card sorting task followed by the declarative medical knowledge rating task. Participants would then be assigned to either the parts or systems interface in a counterbalance order. All participants first read the instruction page of the experiment (see Figure 1a), and then the first task problem would be presented (Figure 1b). Participants then clicked on "Begin" to start the task, which would take them to the main categories page. They could use the "Back" button anytime to go back to the previous page, or click on "View Task" on the main page to review the task problem. Participant could go back to the main page and click on "Done" button when they finished one problem. Participants had to finish one problem in 10 minutes, otherwise, they would be informed that time was up and they had to proceed to the next problem. After the six tasks, participants were asked to do another card-sorting task. The whole experiment took about 2 hours. 


\section{RESULTS}

We performed different statistical analyses to investigate the combined influences of age, interfaces and tasks on three major classes of dependent variables: search performance, search process, and change in knowledge structures. The variables are summarized in Table 1.

\begin{tabular}{|c|l|}
\hline Variables & \multicolumn{1}{c|}{$\begin{array}{c}\text { Description (IV/DV: } \\
\text { independent/dependent variable) }\end{array}$} \\
\hline IV: Age & $\begin{array}{l}\text { Younger participants (Age from18 to 28); } \\
\text { older participants (Age>=60) }\end{array}$ \\
\hline $\begin{array}{c}\text { IV: } \\
\text { Interface }\end{array}$ & Parts interface; systems interface \\
\hline IV: Task & $\begin{array}{l}\text { Matched task (ex. Doing parts type tasks in the } \\
\text { parts interface); mismatched task (ex. Doing } \\
\text { parts type tasks in the systems interface) }\end{array}$ \\
\hline $\begin{array}{c}\text { DV: Search } \\
\text { behavior }\end{array}$ & $\begin{array}{l}\text { Number of categories clicked, link decision } \\
\text { time, broadness, comprehension time, within- } \\
\text { category index }\end{array}$ \\
\hline $\begin{array}{c}\text { DV: Search } \\
\text { performance }\end{array}$ & $\begin{array}{l}\text { Likelihood judgment ratings, accuracy of } \\
\text { multiple choice questions }\end{array}$ \\
\hline $\begin{array}{c}\text { DV: } \text { Mental- } \\
\text { interface } \\
\text { match index }\end{array}$ & $\begin{array}{l}\text { Transformed correlation coefficients between } \\
\text { before/after card sorting data and the } \\
\text { parts/systems interfaces respectively }\end{array}$ \\
\hline
\end{tabular}

Table 1. Summary of variables

\section{Analysis on Background Medical Knowledge}

For the medical declarative knowledge, ANOVA test showed that there was an age effect on their accuracy of prevalence rates $(F(1,45)=10.74, p<.01)$, which implied that older participants had more accurate medical knowledge than younger participants. The result was consistent with the previous study [2].

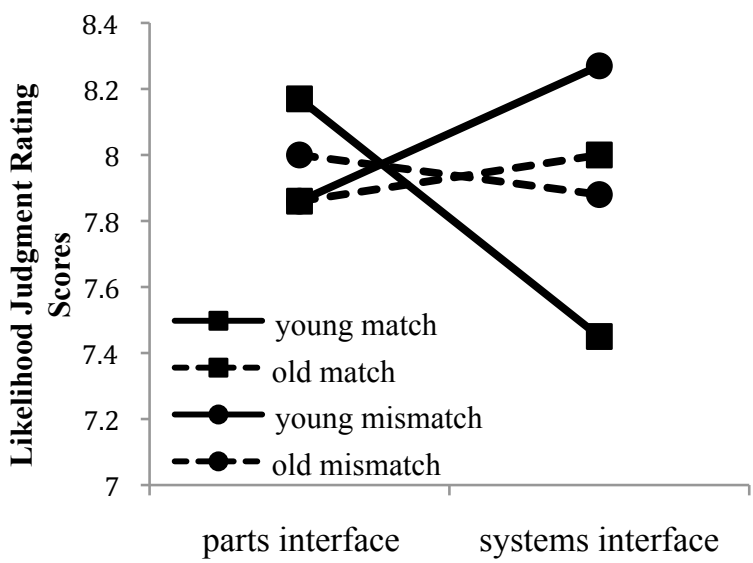

Figure 2. Likelihood judgment rating scores for participants with matching and mismatching tasks and interfaces. The three-way interaction was significant.
Analysis on Search Performance

Effects of Age and Interface on Search Performance

A three-way repeated measures analysis of variance was performed with task, interface, and age as independent variables on their search performance measures, which included the scores of likelihood judgment ratings and accuracy of multiple-choice questions. There were no main effects of age or interface on their scores of likelihood judgment ratings and the accuracy of multiple choice knowledge questions. However, there was a three-way interaction between task, age and interface on the likelihood judgment ratings $(F(1,42)=4.16, p<.05)$ (See Figure 2$)$.

To understand the three-way interaction, we did two twoway ANOVA by different age groups separately. We found that there was no interaction between task and interface on performance of older adults. The three-way interaction could therefore be attributed to the significant interaction between task and interface on likelihood judgment ratings of younger adults $(F(1,21)=5.08, p<.05)$. In other words, older adults' judgment performance remained more consistent in both matched and mismatched tasks regardless of the interfaces; younger adults' judgment performance, however, varied according to the tasks and interfaces. Specifically, when performing a system task in the systems interface, younger participants performed relatively worse, but when performing a parts task in the systems interface, younger adults performed better. This difference was reversed when younger participants were in the parts interface. The results provided support to the intuitive notion that there was an asymmetry between transforming description of body parts to functional body systems and vice versa. This asymmetry of translating different task description to different interfaces in two age groups would be elaborated below.

\section{Analysis on Search Process}

Since the judgment performance of younger and older adults interacted with tasks and interfaces differently, we found that their search processes also depended on the interaction between types of tasks and interfaces. During the task, the time people spent on each page and the links clicked were recorded. To better characterize the search process, we created some search behavior variables in our analysis.

\section{Dependent Measures}

Broadness measured the total links people clicked in the main category and subcategories pages, which referred to how broad people browsed in one task. Link decision time measured the average time people spent deciding on a link click, which reflected the time it took for participants to select a link on a page. Comprehension time measured the average time people spent reading the disease descriptions in the final webpage.

Based on previous research, we expected that younger participants would tend to use a more bottom-up interfacedriven strategy and older participants would use a more topdown knowledge-driven search strategy. One implication 
was that younger participants would more likely follow the interface layout and sequentially click on links grouped under the same category; while older participants would less likely follow the interface layout, but rather rely on the background knowledge to decide which link to click. If this were true, then we would see more within-category consecutive link clicks for younger participants, and more between-category consecutive link clicks for older participants. To verify this claim, we calculated an index that we called the within-category index, which was calculated by dividing the number of links clicked on the symptoms page by the number of links clicked on the main category page. A high within-category index implied that participants clicked more links within any main category.

\section{Age Difference on Search Behavior}

We performed several repeated measure analyses of variance (ANOVA) with age, interface, and task being independent variables on each of the dependent measures. We consistently observed significant main effects of age on broadness, link decision time and comprehension time $(F(1,42)=16.84 ; \quad F(1,42)=32.16 ; \quad F(1,42)=27.11$, respectively; all $p$ 's $<0.001)$. We found that older participants clicked on fewer links on the main category and symptoms pages, spent more time deciding on links, and more time comprehending the disease description than younger participants.

\section{Interaction of Age and Interface on Search Process}

To better understand how interfaces influenced the search behavior of younger and older participants, we did other three repeated measures ANOVA with age, interface, task (match/mismatch between tasks and interfaces) as the independent variables on the number of categories clicked, within-category index and the link decision time on the main category page. For the number of categories clicked, we found a significant main effect of task $(F(1,42)=23.26$, $p<0.001)$, with participants clicked on fewer categories when the tasks matched the interfaces than when there was a mismatch (see Figure 3a). In other words, when participants were able to find matching description between the task and the interface, they tended to focus on the matching category (either parts or systems).

The interface $\mathrm{x}$ age interaction on the number of categories clicked was significant $(F(1,42)=4.03, p<0.05)$. As Figure 3 a shows, younger participants clicked on more categories in the parts interface than in the systems interface $(p<0.05)$, but for older participants the number of categories clicked in the two interfaces were about the same. This was consistent with the notion that younger participants tended to adopt different strategies in different conditions, but older adults tended to adopt the same strategies.

Because the three-way interaction was not significant, we then did two separate repeated measures ANOVA in the two age groups to understand the effect of interface and task on the number of categories clicked. For younger adults, in addition to the main effect of task, we found there was a significant main effect of interface $(F(1,21)=6.62$, $p<0.05)$. However, for older adults, there was only a main effect of task without any significant effect from the two interfaces. Thus, beyond the tendency that participants clicked more categories if the task mismatched with the interface, younger adults was influenced more by the interface layouts. For the mismatched tasks, younger adults
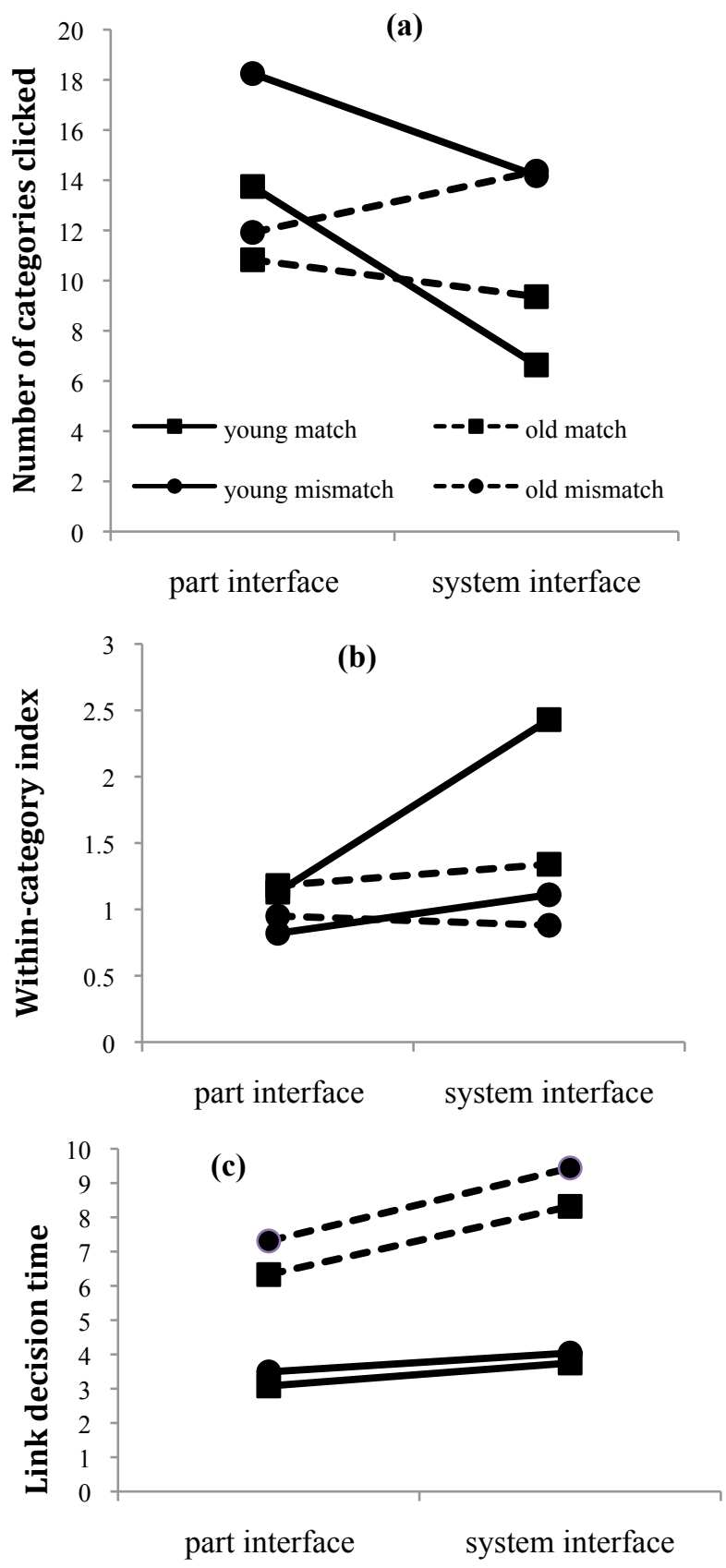

Figure 3. (a) Number of categories clicked, (b) within-category index, and (c) link decision time for younger and older participants when there was a match or mismatch between the task and the interface.

suffered more in the parts interface, suggesting that when given a task with body systems as description in the parts 
interface, the number of categories clicked was larger. This was showing for younger adults it was harder to locate a body part category when a body system description was given (compared to the case when one had to locate a body system category when a body part description was given). This partially reflected the fact that the retrieval strategy and the mental model younger adults used would induce an inherent asymmetry in the knowledge required: Converting from body systems to body parts might be harder than the reverse. For the matched tasks, younger adults clicked more categories in the parts interface to explore the symptoms than the systems interface. Thus, the limited exploration on the matched tasks in systems interface might lead to a suboptimal performance on their likelihood judgment; thus, we found younger adults performed worse in the systemtype tasks with systems interface.

Repeated measure ANOVA with age, task, and interface as independent variables and the within-category index as the dependent variable showed a significant main effect of age on the index, with older participants clicked more on symptoms in different categories, while younger participants clicked more symptoms within the same category $(F(1,42)=6.82, p<0.05)$. There was a significant two-way interaction of task and interface as well as age and interface on the index $(F(1,42)=21.80, \quad p<0.001$; $F(1,42)=11.76, p<0.001$, respectively). And there was a marginally significant three-way interaction of age, task and interface $(F(1,42)=3.48, p=0.06)$. (See Figure $3 b)$. The results suggested that older and younger participants clicked more symptoms within a category when the tasks matched the interfaces. However, the difference between task-interface match and mismatch was significantly larger for younger than older participants.

We did two repeated measures ANOVA on within-category index with task and interface being the independent variables in two age groups separately. For younger adults, in addition to the consistent main effect of task $(F(1,21)=16.41, p<0.001)$, we also found a main effect of interface and a significant interaction effect of task and interface $(F(1,21)=27.36, F(1,21)=6.34$, all $p$ ' $s<0.05)$. For older adults, there was only a main effect of task with more within-category links clicked when the tasks matched with the interface. From the Figure $3 \mathrm{~b}$, we found that older adults searched roughly the same way across different interfaces. But younger adults tended to stick to a few categories in the matched tasks in the systems interface, which was likely the reason for their inferior judgment performance in these tasks. Therefore, only younger adults had varieties of search strategies while encountering different interfaces, especially when the task matched with the interface.

The pattern of results again supported the notion that the search process of older participants was less influenced by the interface than younger participants. Although older participants were adaptive to the different tasks matched or mismatched with interfaces during the search, they tended to use the same top-down strategy in different interfaces. In contrast, younger participants had a higher tendency to adopt different search strategies in different tasks and interfaces. The particularly narrower search patterns of systems tasks in systems interface might be attributed to the higher exploration costs in this specific interface.

Repeated measure ANOVA with age, task and interface as the independent variables and link decision time as dependent variable showed that there were significant main effects of age and task $(F(1,42)=30.28, p<0.001$ and $F(1$, $42)=4.55, p<0.05$ ) (see Figure $3 c$ ). None of the interactions were significant. The results suggested that younger participants spent less time deciding on which links to click, and that for both older and younger participants, the link decision time was shorter when the tasks matched the interfaces.

To summarize, previous research suggested that younger participants used a more interface-driven bottom-up search strategy and older participants used a more knowledgedriven top-down search strategy. In the current study, we still found the same pattern of strategy use. Younger adults tended to look up more links, leave a web page quickly and browse more within-category links. Older adults tended to look up only a subset of links, take longer time to click a link and leave a web page, and browse more betweencategory links. In addition to the age-dependent strategy selection as discussed above, we also found the evidence that strategies adopted by younger participants seemed to have a higher tendency to be influenced by the interface layout as well as the match between their tasks and the interface. In contrast, strategies adopted by older adults tended to be more stable across different combination of tasks and interfaces. Therefore, even if younger adults tended to look up more links and more between-category links than older adults, the age difference became more salient in the in the systems interface. Thus, there were more variances in the search strategies adopted by younger adults in different combination of interfaces and tasks than older adults.

\section{Analysis on Knowledge Change}

From our card-sorting procedures, every participant had two Mental-Interface Match Indexes (MIMI) before and after the tasks. The MIMI reflected how similar participants' mental category structures matched those imposed by the interface. All indices were converted to zscores using Fisher's z-transformation before our analysis to correct for skewness. ANOVA tests showed that there was no significant difference between age groups or interface on the index before the tasks. Thus, before the search tasks, older participants and younger participants had roughly the same match between their mental category structures and those in the interfaces.

We did two separate repeated measure ANOVAs for each interface, with age and before/after task as the independent variables, and MIMI as the dependent variable. The main 
effect of age was not significant. As Figure 4 shows, the index (correlations between mental categories with those in the interface) was fairly low in the parts interface. Given that the parts interface was designed to be an intuitive interface that allowed participants to associate the body part description with the categories without much medical knowledge, the results were consistent with our expectation that participants' knowledge structures did not change much to conform to the categories imposed by the interface.

In the systems interface, repeated measure ANOVA showed a significant interaction effect between age and before/after $(F(1,18)=5.793, p<0.05)$ (See Figure 4). We then did the post-hoc test to test the interaction effect. We found the knowledge structures of younger participants became more similar with the systems interface after the task in terms of gaining higher correlations between participants' knowledge structures and interface's semantic structure after the search $(\mathrm{t}(10)=-2.14, p=0.05)$, but the knowledge structures of older adults didn't have significant difference after the search. Thus, we claimed that younger adults, who adopted more interface-driven strategies, would gain more influence from the type of interface, which induced more demands on the users' top-down resources, such as the systems interface in our study. And for this kind of interface, the internal knowledge structure of younger adults would change towards the external knowledge structure of the interface. However, older adults, who adopted more top-down knowledge-driven strategies, were less likely to generate different search patterns across interfaces. Since they tended to adapt to the different tasks only in their head, their knowledge structure remained the same after the search.

To summarize, compared to strategies adopted by older participants, strategies adopted by younger participants were influenced more by the different search environments created by different combination of task description and interface layouts. But we also found that their knowledge structures were influenced more after the interaction with the interfaces than older adults. Results were consistent with the notion that older adults tended to be less influenced by changes in the task environments, and tended to adopt stable, top-down strategies in different situations.

\section{DISCUSSION}

Our results showed that younger adults tended to use different strategies but older adults tended to use similar strategies to search when engaged in different combination of tasks and interfaces. Specifically, in the interface that required more top-down resources (i.e., in the systems interface), knowledge structures of younger adults were changed to become closer to the structures of the interface after the search task. However, older adults seemed to have relatively stable search performance, search strategies, and knowledge structures when interacting with two different interfaces across the matched and mismatched tasks.

To answer our research questions, first we did find

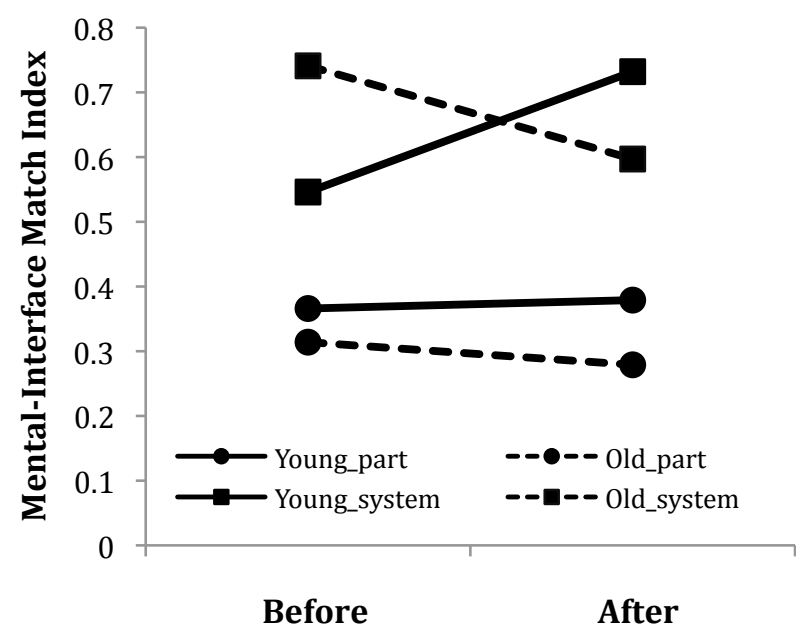

Figure 4. The Mental-Interface Match Index for younger and older participants in the parts and systems interfaces before and after the tasks.

consistent age difference on search strategies. While younger adults used more bottom-up interface-driven search strategies (such as shorter link decision time, higher within-category index and more categories clicked); older adults used more top-down knowledge-driven strategies (such as longer link decision time, lower within-category index and fewer categories clicked). Second, both age groups changed their search patterns according to the matched or mismatched tasks, but with significantly bigger effects in younger adults. The bigger difference in younger adults might have come from the inherent asymmetry of the processes of converting items to different organization (parts to systems was different from systems to parts). Third, the knowledge structures of younger adults were changed in the systems interface, but older adults were found to be more resilient in their knowledge structures.

The natural question was: Given that we did not find any main effect of interface on either search performance or search process of older adults, does this imply they were less adaptive to the different task environments? We would argue that the answer to this question would be a "No". The major reason was that we believe that the stabilized strategy across different interfaces of older adults did not translate to less adaptation. First, according to previous studies [10, 22, 24], the cost of using a different strategy (in this case a more bottom-up search strategy) was higher than its benefit. Because we believe that older participants had better background medical knowledge, the cost of transforming description from parts to systems or vice versa would be lower than those for younger participants. In fact, adopting a bottom-up strategy would have incurred higher costs because of their generally lower perceptual-motor and processing speeds.

From a slightly different perspective, given that the major difference between the interfaces was the different presentation structures of the same information, assuming 
that there were no difference in effort for participants to transform the task description to match the interface, the number of link clicks would have been the same. In other words, most of the difficulties introduced in the unmatched conditions were caused by the mental transformation of medical terms. Assuming that younger participants might have found the transformation more difficult, their switch to a bottom-up strategy (by clicking more categories until the correct one was found) would allow them to reduce the mental effort required for the transformation. But this benefit would not be as strong for older adults, because presumably they had more experience in transforming between those terms. Therefore, so long as there was no considerable change in cost structures between the two interfaces, older adults were actually more adaptive to use the same strategy. Indeed, when we look at older participants' search process and performance in mismatched tasks, there was no difference in two interfaces. It was possible that older adults might have a more dedicate, complicated or abundant semantic structure of medical domain knowledge than those of younger adults.

Results from our study showed that the dynamic interactions between age, interface and task on search strategies and performance. Consistent with previous findings, we found that knowledge structures played an important role on the search process in terms of inducing possible mental transformation costs between mismatch of medical symptom description between the task and the interface, which in turn lead to the use of different search strategies.

\section{REFERENCES}

1. Anderson, J.R. The Adaptive Character of Thoughts. Hillsdale, Erlbaum, NJ, USA, 1990.

2. Beier, M.E. and Ackerman, P.L. Determinants of Health Knowledge: An Investigation of Age, Gender, Abilities, Personality, and Interests. Journal of Personality and Social Psychology, 84, (2003), 439-448.

3. Brumby, D.P. and Howes, A. Strategies for Guiding Interactive Search: An Empirical Investigation into the Consequences of Label Relevance for Assessment and Selection. Human-Computer Interaction, 23, (2008), 1-46.

4. Chin, J., Fu, W-T. and Kannampallil, T. Adaptive information search: Age-dependent interactions between cognitive profiles and strategies. In Proc. CHI2009, ACM Press (2009), 16831692.

5. Charman, S.C. and Howes, A. The adaptive user: An investigation into the cognitive and task constraints on the generation of new methods. Journal of Experimental Psychology: Applied, 9, (2003), 236-248.

6. Czaja, S.J., Sharit, J., Ownby, R., Roth, D. and Nair, S. Examining Age Differences in Performance of a Complex Information Search and Retrieval Task. Psychology and Aging, 16, (2001), 564-579.

7. Fairweather, P. How Older and Younger Adults Differ in Their Approach to Problem Solving on a Complex Website. In Proc. ASSETS2008, ACM Press (2008), 67-72.
8. Fisk, A.D., Rogers, W.A., Charness, N., Czaja, S.J., and Sharit, J. Designing for Older Adults-Principles and Creative Human Factors Approaches. CRC Press, Washington, DC, USA, 2004.

9. Flynn, K.E., Smith, M.A., and Freese, J. When do older adults turn to the internet for health information? Findings from the Wisconsin longitudinal study. Journal Gen Intern Med, 21, (2006), 1295-1301.

10. Fu, W.-T., and Gray, W.D. Suboptimal Tradeoffs in Information-Seeking. Cognitive Psychology, 52, (2006), 195242.

11. Fu, W.-T., Pirolli, P. SNIF-ACT: A cognitive model of user navigation on the World Wide Web. Human-Computer Interaction, 22, (2007), 355-412.

12. Hutchins, E. Cognition in the Wild. MIT Press, Cambridge, MA, USA, 1994.

13. Kirsh, D., and Maglio, P. On distinguishing epistemic from pragmatic action. Cognitive Science, 18, (1994), 513-549.

14. Mata, R., Schooler, L. J. and Rieskamp, J. The aging decision maker: cognitive aging and the adaptive selection of decision strategies. Psychology and Aging, 22, (2007), 796-810.

15. Meyer, B.J.F., Talbot, A.P. and Ranalli, C. Why older adults make more immediate treatment decisions about breast cancer than younger adults. Psychology and Aging, 22, (2007), 505524 .

16. Miller, L.M.S., Stine-Morrow, E.A.L., Kirkorian, H.L. and Conroy, M.L. Adult age differences in knowledge-driven reading. Journal of Educational Psychology, 96, (2004), 811821.

17. Payne, S.J., Howes, A. and Reader, W.R. Adaptively distributed cognition: a decision making perspective on human-computer interaction. Behaviour \& Information Technology, 20, (2001), 339-346.

18. Pew Internet \& American Life Project. (2008). The Engaged E-patient Population. [Online]. Available: http://www.pewinternet.org/pdfs/PIP_Health_Aug08.pdf

19. Simon, H.A, Rational choice and the structure of the environment. Psychology Review, 63, (1956), 129-138.

20. Simon, H.A., The Structure of Ill-Structured Problems. Artificial Intelligence, 4, (1973), 181-204.

21. Sharit, J., Hernandez, M., Czaja, S. and Pirolli, P. Investigating the Roles of Knowledge and Cognitive Abilities in Older Adult Information Seeking on the Web. ACM Transactions on Computer-Human Interaction, 15, (2008), 1125.

22. Stine-Morrow, E.A.L., Miller, L.M.S., Gagne, D.D. and Hertzog, C. Self-Regulated Reading in Adulthood. Psychology and Aging, 23, (2008), 23, 131-153.

23. Stronge, A.J., Rogers, W.A. and Fisk, A.D. Web-based information search and retrieval: effects of strategy use and age on search success, Human Factors, 48, (2006), 434-446.

24. Touron, D. R., and Hertzog, C. Strategy shift affordance and strategy choice in young and older adults. Memory \& Cognition, 32, (2004), 298-310. 